\title{
Knowledge, Attitude and Practice of Postmenopausal Osteoporosis among Females Aged 17-24 Years, Khartoum, Sudan
}

\author{
Article by Shahd Khalid Mohammed El Khalil ${ }^{1}$ \\ ${ }^{1}$ MBBS \\ Zeinab Swareldahab ${ }^{2}$ \\ ${ }^{2} \mathrm{MBBS}, \mathrm{MD}$ \\ Osama A. A. Elkhidir ${ }^{3}$ \\ ${ }^{3}$ MBBS, MPH, Diploma health economics \\ Faculty of Medicine, University of Khartoum \\ E-mail: zeinabswar@hotmail.com
}

\begin{abstract}
Background: Osteoporosis (OP), a metabolic disorder which affects mainly bones, is characterized by low bone mass and is associated with serious health and economic burden. The main musculoskeletal problems associated with it are hip fractures with a worldwide prevalence of about 1.6 million and high rates of morbidity and mortality. It is a silent disease and usually does not manifest until fractures occur. The Aim of this study were to assess knowledge, attitude and practice toward postmenopausal osteoporosis and its preventive measures among female students aged 1724years in Khartoum, Sudan.

Methodology: A cross sectional study was conducted in three different faculties of the University of Khartoum. Data was collected from 226 female students aged 17-24 by stratified systematic random sampling using a pre-tested, pre-coded self-administered questionnaire.

Results: The majority (84.8\%) of respondents heard about osteoporosis. Faculty curriculum was the main source of information (31.1\%), followed by family and friends (15.3\%). The overall knowledge of respondents about OP was below average (57.1\%), average (42\%) and good (0.9\%). About three-fifth (58.1\%) were aware about their susceptibility to get it in the future and (61\%) were concerned about taking preventive measures. The majority of respondents (94.9\%) perceived the seriousness of OP. Milk and dairy products were the most commonly identified as sources of calcium (33.8\%).

Conclusions: The overall knowledge was below average. Raising knowledge about osteoporosis may be an effective tool in preventing the problem and subsequently osteoporotic fractures later in life.
\end{abstract}

Keywords: postmenopausal oosteoporosis, hip fractures, knowledge, attitudes, practice.

\section{Introduction}

Osteoporosis (OP) is an acquired disease characterized by reduced bone mass and deterioration of the micro-architectural structure of bone tissue, leading to increased bone fragility and susceptibility to fractures (Kumar et al; 2013). World Health Organization (WHO) defines osteoporosis as a bone mineral density of 2.5 standard deviations or more below the mean peak bone mass as measured by dual-energy X-ray Absorptiometry (DEXA scan) (Dontas et al; 2014).

Bone loss in osteoporosis may be confined to certain bones and regions or to be generalized. Generalized OP may occur as a primary disease or secondary to a variety of diseases including metabolic diseases, vitamin deficiencies and drug exposure. Primary OP is the most common form and may be associated with aging. In both men and women OP begins in the third or fourth decade of life when a dynamic balance between bone formation and bone resorption tilt in favour of resorption the bone loss averaging $0.5 \%$ per year occurs mostly in areas containing the abundant trabecular bone. The decline in oestrogen levels associated with the menopause in females correlate with an acceleration of cortical and trabecular bone loss. Over 30 to 40 years this can result in loss of up to $35 \%$ of cortical bone and $50 \%$ of trabecular bone which leads to postmenopausal women suffering 
from osteoporotic fractures. It appears that the post-menopausal drop in oestrogen leads to increased cytokines production this stimulate (RANK-RANK Ligand) activity and suppress Osteoprotegrin (OPG) production that increases osteoblastic activity. The risk of primary osteoporosis is related to peak bone mass earlier in life which is influenced by genetic, nutritional and environmental factors (Dalsky et al; 1988).The disease is usually manifested clinically as fracture that affects and burdens the hip, vertebral column, wrist and other peripheral bones. Diagnosis can be made using radiological studies and by measuring bone mineral density by dual energy x-ray absorptiometry.

Primary OP can be prevented by lifestyle modifications, use of certain medications or both. Life style modification includes education of the people and persuading them to do regular exercise, reduces weight, take a balanced and healthy diet and quit smoking and alcohol (Dalsky et al; 1988). Calcium and vitamin D supplements are used in high risk individuals other drugs include oestrogen replacement therapy for prevention of OP but it is not recommended unless there are other indications for its use because of its associated side effects (Kumar et al ;2013). Worldwide, OP causes more than 8.9 million fractures annually or an osteoporotic fracture every 3 seconds (Johnell et al; 2006). According to the International Osteoporosis Foundation, OP is estimated to affect 200 million women worldwide. Although OP is a silent disease it can be prevented, early diagnosed and treated. The level of awareness about the disease's risk factors and preventive measures is an important factor in decreasing osteoporotic fractures. There is observable increase in osteoporotic fractures among postmenopausal females in low income countries and the Middle East (Morales et al; 2007).

A cross sectional study was conducted in 2013, to evaluate KAP of OP among male and female students in a Malaysian university. The research reported higher levels of knowledge among females compared to males (Y.H.Khan et al; 2014). Another study in Pakistani female university students concluded that, about (82.1) percent of participants had good knowledge about the disease (Noman-ulHaq et al 2015). The awareness level of Indian female students regarding the disease was low (Mukesh et al; 2007). A campaign among King Abdul-Aziz University students in the Kingdom of Saudi Arabia (KSA) showed that the majority of students (77\%) had high scores of knowledge about the disease (Ghadeer and Haytham; 2015).

OP was not integrated in the educational curricula in the majority of Arab countries (Dontas et al; 2007). A more focused survey involved 353 female nursing students in Damascus identified general knowledge about OP as poor and that it was not included in the faculty curriculum (Rima et al;2013).

A cross sectional study in the KSA targeting adult women showed higher levels of knowledge among the young, those of low socioeconomic status and housewives, while more than half $(55 \%)$ of respondents showed good attitude toward OP. In practice, however, only (18.2) percent were exposed to sunlight, (35.4) percent performed exercise, (41.5) percent consumed soft drinks and (50.8) percent ate calcium rich foods (Kh. Eltohami et al; 2015). A KAP survey done in Kerman, Iran indicated insufficient knowledge, adequate protective exercise and insufficient calcium intake (Z. Jalili et al; 2007). An Egyptian study revealed poor knowledge and attitude with no differences between males and female (Hala et al; 2015). In a further study in Iran the mean awareness score was 11.7 from an overall score of 19, it was reported as not good and significantly higher levels knowledge was found among participants with higher educational levels (MansourehSafizadeh et al; 2015).

Hip fractures in the Middle East and Africa fall in the lower third of the scale worldwide. Only about $0.98 \%$ of the global publications about OP were from Arab countries (waleed et al; 2014).

\section{Objectives}

The general objective was to study the knowledge, attitude and practice (KAP) toward postmenopausal OP among female university students aged 17-22.

The specific objectives were to:

1. Assess knowledge about postmenopausal OP

2. Explore attitudes toward preventing it.

3. Identify practice of preventive measures 


\section{Methodology}

\section{Research design}

Descriptive cross-sectional study.

\section{Study area}

Faculties of Medicine, Nursing and General Environmental Health of the University of Khartoum.

\section{Study population}

total population was 2149 female students.

Inclusion criteria were Sudanese female students, aged 17-22 that formally registered. Exclusion criteria were those who refused to participate in the study.

\section{Sample size}

Up to our knowledge there was no published data about prevalence of OP in Sudan, hence, $28.4 \%$ of neighbourhood country (Egypt) was used (IOF; 2011).

It was calculated according to the equation:

$\mathrm{n}=\frac{\mathrm{N} \mathrm{z}^{2} \mathrm{p}(1-\mathrm{p})}{\mathrm{N} \mathrm{d}^{2}+\mathrm{z}^{2} \mathrm{p}(1-\mathrm{p})}$ Where:

$\mathrm{n}=$ sample size

$\mathrm{N}=$ total number of population (2149)

$\mathrm{z}=\mathrm{z}$ value (e.g. 1.96 for $95 \%$ confidence level)

$\mathrm{d}=$ Desired margin of error, expressed as decimal (0.05)

$\mathrm{P}=$ Prevalence of the disease $(28 \%)$

$\mathrm{n}=270$

Only 226 students were responded

\section{Sampling selection technique}

Stratified systematic random proportional to the number of students in each faculty and academic year was used. Each faculty was considered as stratum. Proportional to the number of female students in the 3 faculties, the appropriate number of students was assigned to each faculty and accordingly to each academic year using the formula: $\mathrm{ni}=\frac{\mathrm{Ni}}{\mathrm{N}} * \mathrm{n}$

Students from each class were selected by systematic random sampling in both medicine and nursing faculties. However, students from faculty of general health were selected conveniently due to the unavailability of student list during the time of study.

\section{Data collection tool}

Pre-tested, pre-coded self-administered questionnaire modified from the Malaysian Osteoporosis Prevention and Awareness Tool (OPAAT) composed of 4 parts (Toh LS et al; 2015).

\section{Data management}

Data was analysed using SPSS version 21. Percentages were computed. Chi square test was used to assess difference between proportions. A scoring system was used by giving correct answers one mark.

\section{Ethical consideration}

Ethical clearance was obtained from the Faculty of Medicine, University of Khartoum Research Committee. Verbal consent was obtained from each participant following clarification that the confidentiality of the data would be observed. 
DOI: $10.21522 /$ TIJPH.2013.06.03.Art017

ISSN: $2520-3134$

\section{Results}

\section{Background data}

Two hundred and twenty six students responded to the questionnaire. A majority of (70\%) were studying medicine, $(12.4 \%)$ nursing and (17.3\%) were environmental health students. About two third (64.4\%) were during their intermediate and post intermediate academic years (second to fourth years). The overwhelming majority (97.8\%) were single however; participants were almost equally distributed according to the economic status, family income-per month in SDG; (55.5\%) less than 4000 and (44.4\%) 4000 and more (table 1).

Table 1. Distribution of respondents according to background data.

\begin{tabular}{llll}
\hline 1. & Faculty & $\mathbf{n}$ & $\mathbf{\%}$ \\
\hline Medicine & 159 & 70.3 \\
Nursing & 28 & 12.4 \\
& Environmental Health & 39 & 17.3 \\
\hline Total & $\mathbf{2 2 6}$ & $\mathbf{1 0 0 . 0}$ \\
\hline $\mathbf{2 .}$ & Academic year & & \\
& Year 1 & 25 & 11.1 \\
& Year 2 & 50 & 22.2 \\
& Year 3 & 46 & 20.4 \\
& Year 4 & 49 & 21.8 \\
& Year 5 & 42 & 18.7 \\
& Year 6 & 13 & 5.8 \\
\hline & Total & $\mathbf{2 2 6}$ & $\mathbf{1 0 0 . 0}$ \\
\hline 3. & Marital status & & \\
& Single & 221 & $\mathbf{9 7 . 8}$ \\
& Married & 5 & $\mathbf{2 . 2}$ \\
\hline & Total & $\mathbf{2 2 6}$ & $\mathbf{1 0 0 . 0}$ \\
\hline 4. & Family income/month & & \\
& in SDG & & \\
& $<2,000$ & $\mathbf{3 7}$ & 22.8 \\
& 2,000-<4,000 & $\mathbf{5 3}$ & 32.7 \\
& 4,000 and more & $\mathbf{7 2}$ & 44.4 \\
\hline & Total & $* 162$ & $\mathbf{9 9 . 9}$ \\
\hline
\end{tabular}

*64 students did not answer this question about family income 


\section{Sources of information}

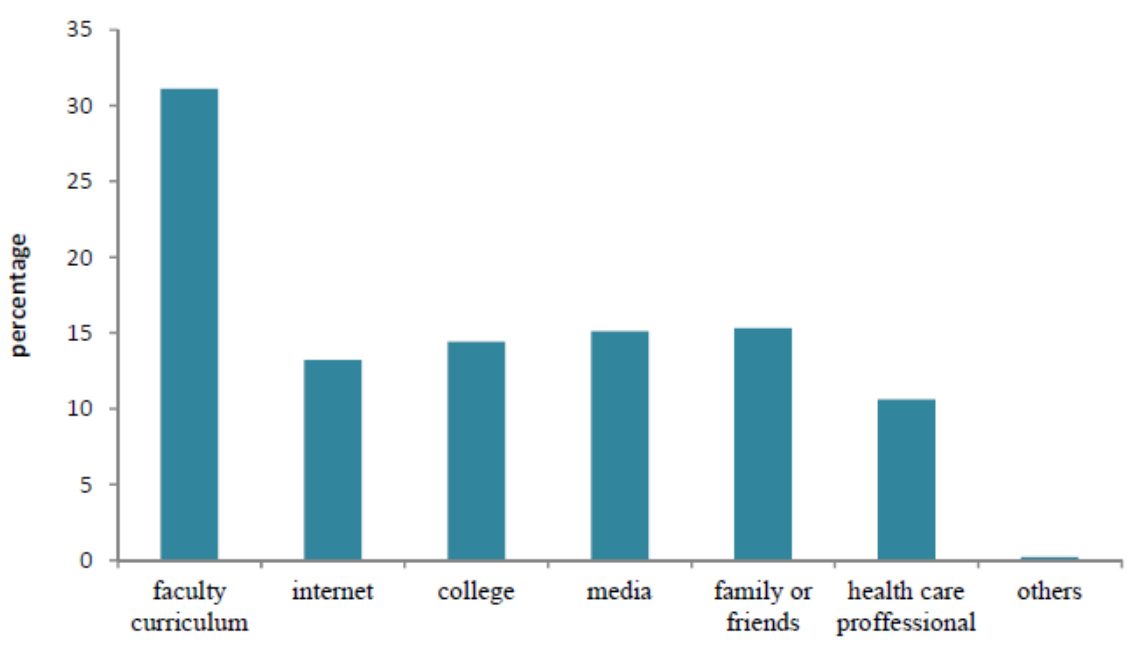

Figure 1. Sources of information of respondents who heard about OP

Figure 1 shows sources of information of respondents who heard about OP. Faculty curriculum was the most common source (31\%), then family/friends, college, media, internet and health professional (about $10-15 \%$ each).

\section{Correct knowledge}

Table 2 shows the distribution of respondents by their scores of correct knowledge of OP. Scores ranged between 84.5 percent for, OP making bones weaker, brittle and more likely to break causing fractures to 9.7 percent for untreated OP resulting in loss of mobility.

Table 2. Distribution of respondents by their scores of correct knowledge of OP.

\begin{tabular}{lll}
\hline No & Question on knowledge of OP & Correct response \\
\cline { 2 - 3 } & & $\mathrm{n}$ \\
\hline
\end{tabular}

1. Makes bones weaker, brittle and more likely to break (fractures) 191

2. Everybody will get osteoporosis as it is part of aging.

$88 \quad 38.9$

3. The probability of osteoporosis is equal in men and women. 139

4. Post-menopausal women are not at risk for osteoporosis. 157

61.5

5. Osteoporosis usually has no symptoms

6. Consumption of cortisone for $\geq$ three months can lead to osteoporosis.

7. Genetic factors have no relationship with osteoporosis. 105

8. Being underweight helps prevent osteoporosis.

9. Weight bearing exercise (e.g. jogging) decrease bone loss.

10. Smoking increases the risk of osteoporosis.

11. Osteoporosis is an untreatable disease

12. A bone mineral density test is used to diagnose osteoporosis.

13. Premenopausal ( $<45$ years) are more likely to get osteoporosis

14. The skeleton riches its maximum strength at 20sYears of age.

15. Untreated osteoporosis results in back pain

16. Untreated osteoporosis results in loss of height or hunchback

17. Untreated osteoporosis results in joint pain or swelling of

18. Untreated osteoporosis results in Hip fractures

19. Untreated osteoporosis results in loss of mobility 
DOI: $10.21522 /$ TIJPH.2013.06.03.Art017

ISSN: $2520-3134$

21. There is no way to prevent osteoporosis

22. Recommended daily amount of ca. increases in females after 50

23. It is too late to increase calcium intake after the age of 50 .

24. Calcium supplement helps in prevention of osteoporosis.

25. The regular dose of calcium supplements can cause kidney

26. Daily intake of vitamin $\mathrm{D}$ is obtained by daily exposure to sunlight

27. Decreasing intake of carbonated drinks help in disease prevention.

\section{Overall knowledge}

The overall knowledge of respondents about OP revealed the following ranges of scores:

The total score for each respondent was calculated. A cut of level $(\leq 15)$ was considered as below average knowledge, (16-21) as average knowledge while a total score of $(\geq 22)$ was considered as good. Out of the total score of (27) marks the mean total score of knowledge was calculated $(12.88 \pm 6.23)$.

Knowledge about dietary sources of calcium showed that milk and dairy products were the most commonly identified source (33.8\%), followed by dates (16.6\%), greens vegetables (15.7\%), tuna and sardines (13.7\%). Dried fruits, nuts and sasame were the least identified source, $11.2 \%$ and $9 \%$, respectively.

\section{Attitudes}

The majority of respondents $(94.9 \%)$ perceived the seriousness of OP. About (58\%) were aware about their susptibility to it and $(61 \%)$ were concerned about taking preventive measures. Increasing the dietary intake of calcium (23.9\%) and decreasing the consumption of carbonated drinks $(22.5 \%)$ were the most preferred preventive behaviours.

\section{Practice}

The practice of certain lifestyle habits that affect future risk of osteoporosis shown in

Table 3 Shows the practice of certain lifestyle habits that affect future risk of OP

The practice of preventive behaviours revealed that four fifths $(80.0 \%)$ of respondents consumed calcium and vitamin D rich foods, about one third (31.1\%) performed weight bearing regular exercise and about three fifths (58.4\%) were usually exposed to the sun. The practice of risk behaviours showed that about three quarter (71.8\%) do not consume carbonated drinks, less than one tenth $(8.4 \%)$ were cortisone users and $(0.9 \%)$ were smokers.

Table 3. Practice of certain lifestyle habits that affect future risk of OP

\begin{tabular}{llll}
\hline Behaviour/Life style habit & \multicolumn{2}{c}{ Positive practice } \\
\cline { 3 - 4 } 1. Preventive & 1.1 Calcium intake & 181 & 80.0 \\
& 1.2 Weight bearing & 68 & $(31.1)$ \\
& 1.3 Exposure to sunlight & 129 & $(58.4)$ \\
& $\begin{array}{l}\text { 1.4 Not consuming } \\
\text { carbonated drinks }\end{array}$ & 62 & $(28.2)$ \\
2.1 Cortisone use & 19 & $(8.4)$ \\
& 2.2 Smoking & 2 & $(0.9)$ \\
\hline
\end{tabular}




\section{Discussion}

This study was conducted to assess KAP toward postmenopausal OP among female university students. The young sector of the community is amenable to behavioural change because it is at the age of peak bone mass which positively affects the risk of the disease (Juan et al; 2014) furthermore, university students are catalysts in raising community awareness.

Results revealed that more than half $(57 \%)$ of the respondents had insufficient knowledge about the disease. Similar results were reported among female students in Damascus (Rima et al; 2013). However, good knowledge (82.1\%) about OP was reported among female student in Malaysia (Y. H. Khan; 2014). These results seemed to indicate that the level of knowledge about OP among this sector of the population were contrary to expectations. This might be due to lack of available sources of knowledge for this at risk sector (Ghadeer and Haytham; 2015). The most common source of information about OP was the curriculum, followed by family or friends, while the least was health care professionals, the media and internet. Involvement of health care professionals in delivering information about the disease was noticed among women of older ages in USA (Stacie et al; 2001) and Egypt (Sara et al; 2016).

Being female and the postmenopausal state were the non-modifiable risk factors that known by the respondents, $61.5 \%$ and $69.5 \%$, respectively. This does not agree with other studies which had shown poor knowledge about non-modifiable risk factors (Noman-ul-Haq et al; 2015).

Overall good knowledge about modifiable risk factors was shown by respondents (despite poor knowledge about smoking and regular use of cortisone), higher than that reported in previous studies in Syria (Rima et al; 2013) and Malaysia (Y.H.khan et al; 2014).

It is worthy to note that the age of peak bone mass is more encouraging for adopting preventive practice against OP; but, unfortunately, a considerable number of respondents did not know this. Similar results were observed in Indian females (Mukesh et al; 2103). As vitamin D is one of the important determinants for development of bones during childhood and adolescence (Hreybe et al; 2004), exposure to sunlight is considered important and was assessed in this study. Interestingly about $10.7 \%$ of respondents were never had enough exposure to sunlight as they mentioned which put them at risk of developing hypovitaminosis D and hence OP. On the other hand (57.8\%) of respondents were exposed enough to sunlight this rate is higher than $18.2 \%$ reported in Saudi females (Kh. Eltohami et al; 2015) and lower than $88.2 \%$ reported in Iranian females (Z. Jalili et al; 2007). Exposure of Sudanese females is below expectations as sufficient sun light is available throughout the year in Sudan.

Weight bearing exercises were performed by only one third of our respondents which is higher than that reported by comparable studies (Kh. Eltohami et al; 2015; Z. Jalili et al; 2007).

There was no association observed between the level of knowledge and practice of exercise.

Four fifths $(80.0 \%)$ of respondents consumed calcium and vitamin D rich foods this might be be linked to Sudanese diatry cultural practice rather than knowledge translation. Only about one fifth of respondents had adequate calcium intake. Similarly, a low level was reported in Iran (Morales et al; 2007).This might be attributed to difference in economic and cultural factors such as the lack of interest toward knowing about micronutrient compositions of different types of foods and unavailability of nutritional fact sheets for Sudanese foods.

\subsection{Conclusions}

Overall knowledge was below average and respondents were unaware of the risk factors and Consequences of OP. Raising knowledge may be an effective tool for preventing OP, osteoporotic fractures and other sequels.

\section{Recommendations}

Mass ccampaigns are essential in universities to raise the awareness about the OP. Development of nutritional fact sheets for Sudanese food and make them accessible especially for the for young females who are at the age when preventive measures against OP are most effective. Further research involving wider sectors of the community is required 
DOI: $10.21522 /$ TIJPH.2013.06.03.Art017

ISSN: $2520-3134$

\section{Limitations}

- The response rate of answering the self-administered questionnaire was 56.5 percent because they were busy with studying and doing their clinical hospital based rounds.

- Sixty four (28.3\%) students did not answer the question about family income because they were not directly involved with their families' sources of money.

\section{References}

[1]. Dalsky GP, Stocke KS, Ehsani AA, Slatopolsky E, Lee WC, Birge SJ. Weight-bearing exercise training and lumbar bone mineral content in postmenopausal women. Ann. Intern. Med. 1988;108 (6): 824-8

[2]. Exucutive summary. Middle East and Africa audit. International osteoporosis foundation 2011. A report

[3]. Ghadeer zakai, haytham zakai. Awareness about osteoporosis among students in Jeddah, Saudi Arabia. Journal of Advanced laboratory research in biology 2015; 6.

[4]. Hala M. Elsabagh, Abdelaziz F. Aldeib, Salwa A. Atlam, Shimaa M. Saied. Osteoporosis knowledge and Health Beliefs among Employees of Tanta University. American Journal of Research Communication; 2015: 12.

[5]. Hreybe H, Salamoun M, Badra M, Afeiche N, Baddoura O, Boulos S, Haidar R, Lakkis S, Musharrafieh R, Nsouli A, Taha A, Tayim A, El-Hajj Fuleihan G. Hip fractures in Lebanese patients: determinants and prognosis. J Clin Densitom. 2004; 7(4):368-375.

[6]. I.A. Dontas and C.K. Yiannakopoulos. Risk factors and prevention of osteoporosis-related fractures. J Musculoskelet Neuronal Interact 2007; 7(3):268-272.

[7]. Johnell $\mathrm{O}$ and Kanis JA. An estimate of the worldwide prevalence and disability associated with osteoporotic fractures. OsteoporosInt 2006; 17:1726.

[8]. Juan Lu, Yongyun Shin, Miao-Shan Yen, and Shumei S. Sun. Peak bone mass and patterns of change in total bone mineraldensity and bone mineral contents from childhood into young adulthood. Assessment \& Management of Musculoskeletal Health Journal of Clinical Densitometry: 2014.

[9]. Kh. Eltohami, W.Sami, A. Al eidan, M. Almobarak, F. Alotaibi. Study of knowledge, attitude, and practice of osteoporosis among adult women in Majmaah city, Saudi Arabia. International journal of health and rehabilitation science; 2015:4:3.

[10]. Kumar, Abbas, Aster, Robbins Basic pathology, Ninth ed., Canada, Elsevier 2013;768-770.

[11]. Mansoureh Safizadeh, Elahe Aminizadeh, Hossein Safizadeh. Awareness of osteoporosis among female employees in Kerman, Iran. Russian Open Medical Journal; 2015;4;1

[12]. Morales-Torres J. Strategies for the prevention and control of osteoporosis in developing countries. Clin Rheumatol,2007;26(2):139-143.

[13]. Mukesh Srivastava, Gul Naz Fatima, Richa Srivastava1 and Man Mohan Singh. Assessment of Awareness of Osteoporosis Amongst Unmarried Indian Girl Students Pursuing Undergraduateand Postgraduate Professional Courses..Asian Journal of Oral Health \& Allied Sciences 2; 2013; 3.

[14]. Noman-ul-Haq, Maria Tahir, Qaiser Iqbal and Aqeel Naseem. Exploration of Osteoporosis Knowledge and Perception among Young Women in Quetta, Pakistan. J Osteopor Phys; 2015, 3:3.

[15]. Rima Sayed-Hassan, Hyam Bashour \&Abir Koudsi. Osteoporosis knowledge and attitudes: a crosssectional study among female nursing school students in Damascus. Arch Osteoporos 2013; 8:149.

[16]. Sarah Sayed El-Tawab, Emmanuel Kamal Aziz Saba, Heba Mohmoud Taha Elweshahi, Mona Hamdy Ashry. Knowledge of osteoporosis among women in Alexandria (Egypt): A community based survey. The Egyptian Rheumatologist 2016; 38, 225-231.

[17]. Stacie E. Geller, Richard Derman. Knowledge, beliefs and factors for osteoporosis among African American and Hispanic women. Journal of the national medical association 2001; 93:13-21.

[18]. Toh LS, Lai PSM, Wu DB-C, Wong KT, Low BY, Anderson C. The development and validation of the osteoporosis prevention and awareness tool (OPAAT) in Malaysia. PLoS ONE 2015; 10(5).

[19]. Waleed M Sweileh1, Samah W Al-Jabi, Sa'ed H Zyoud, Ansam F Sawalha1 and Mustafa A Ghanim.Osteoporosis is a neglected health priority in Arab World: a comparative bibliometric analysis; Springer Plus 2014, 3:427

[20]. Y. H Khan, A. Sarriff, A. H Khan, T. H Malhi. KAP survey of osteoporosis among students of tertiary institution in Malaysia. Trop J Pharm Res, 2014;13(1): 155. 
Texila International Journal of Public Health Volume 6, Issue 3, Sep 2018

[21]. Z Jalili, N Nakhae, R Askar, V Sharifi. Knowledge, attitude and preventive practice of women concerning osteoporosis. Iranian J Publ Health, 2007;36, 2:19-24. 